$\begin{array}{lll}\text { Bentham OPEN } & \text { The Open Hypertension Journal } \\ \text { CrossMark } & \text { Content list available at: www.benthamopen.com/TOHYPERJ/ } \\ \text { DOI: } 10.2174 / 1876526201810010028,2018,10,28-40 & \text { The } \\ \text { Open Hypertension } \\ \text { lournal }\end{array}$

RESEARCH ARTICLE

\title{
Nocturnal Blood Pressure Decrease in Hypertensive Patients and Normotensives- Association with Obstructive Sleep Apnoea and Renal Function
}

Bodil G. Hornstrup ${ }^{1,2,}$, Jeppe B. Rosenbaek ${ }^{2}$, Nikolai Hoffmann-Petersen², Pia H. Gjoerup ${ }^{2}$, Jost Wessels $^{2}$, Thomas G. Lauridsen ${ }^{1,2}$, Erling B. Pedersen ${ }^{1}$ and Jesper N. Bech ${ }^{1,2}$

${ }^{I}$ University Clinic of Nephrology and Hypertension, Holstebro Hospital, Aarhus University, Holstebro, Denmark

${ }^{2}$ Department of Medicine, Holstebro Hospital, Holstebro, Denmark

Received: April 19, 2018

Revised: July 26, 2018

Accepted: September 20, 2018

Abstract:

Background:

Blunted nocturnal Blood Pressure (BP) decrease is seen in patients with hypertension and obstructive sleep apnoea (OSA). The influence of OSA and renal function on nocturnal BP decrease is not fully clarified.

\section{Objective:}

In this case control study of hypertensive patients and healthy controls, we aimed to analyse the relationship between nocturnal BP decrease on one hand and presence of OSA, renal function, plasma levels of syndecan and vasoactive hormones, and urinary sodium excretion on the other.

\section{Methods:}

In 75 hypertensive patients and 56 controls, we performed brachial and central $24 \mathrm{~h}$ ambulatory BP measurement and cardio respiratory monitoring. We measured syndecan, renin, angiotensinII, aldosterone, vasopressin, and brain natriuretic peptide in plasma and $24 \mathrm{~h}$ urinary excretion of sodium, aquaporin2, and a component of the epithelial sodium channel (u-ENaC $\gamma$ ).

\section{Results:}

Nocturnal BP decrease was lower in patients than controls, brachial $(13 \%$ versus $17 \%, p=0.001)$ and central $(8 \%$ versus $10 \%$, $p=0.019)$. Moderate-to-severe OSA was present in $13 \%$ of patients, $2 \%$ of controls $(p<0.005)$. Neither brachial nor central nocturnal BP decrease was associated with OSA, renal function, plasma levels of vasoactive hormones, syndecan-1, or urinary sodium excretion. P-syndecan and $\mathrm{u}-\mathrm{ENaC \gamma}$ were higher in patients than controls.

\section{Conclusion:}

Both brachial and central nocturnal BP decrease was lower in patients than in controls. Neither brachial nor central nocturnal BP decrease was associated with the presence of OSA, renal function, or plasma levels of vasoactive hormones. Increased syndecan in plasma in hypertensive patients suggested damage to the endothelial glycocalyx.

Keywords: Nocturnal blood pressure decrease, Hypertension, Obstructive sleep apnoea, Renal function, Central blood pressure, Syndecan.

\footnotetext{
* Address correspondence to this author at the University Clinic of Nephrology and Hypertension, Holstebro Hospital, Aarhus University, Laegaardvej 12J, DK-7500 Holstebro, Denmark, Tel: 457843 6590; E-mail: bodil.hornstrup@rm.dk
} 


\section{INTRODUCTION}

In healthy man, Blood Pressure (BP) shows a circadian rhythm with higher BP during daytime and a nocturnal decrease of $10-20 \%$ of daytime BP [1]. High nocturnal BP or impaired nocturnal BP decrease (non-dipping) are predictors for cardiovascular disease independent of brachial 24h BP [2, 3]. Obstructive Sleep Apnoea (OSA) is associated with high nocturnal BP and non-dipping in both hypertensive patients and in normotensives [4 - 6]. Direct association between severity of OSA and nocturnal BP decrease have only been proven in populations with high probability of or diagnosed with OSA [5, 7]. Yet, the link between OSA and nocturnal BP decrease in hypertensive patients needs to be analysed. High nocturnal BP and non-dipping are often observed in patients with Chronic Kidney Disease (CKD) with increasing incidence as renal function decreases [8 - 10]. No studies are available, in which it has been analysed whether an association exists between nocturnal BP decrease on one hand and mild reduction in renal function and OSA on the other.

Syndecan-1 is a protein component of the endothelial glycocalyx protection layer. Syndecan and other membrane proteins are degraded in vascular diseases and have been used as a marker of cardiovascular stress [11]. A previous study suggested that non-dipping was associated with an abnormal endothelial function [12]. Therefore, it is interesting to determine whether high nocturnal BP and non-dipping are accompanied by an increase in plasma syndecan-1 as a possible indicator of increased endothelial damage.

Central Aortic Systolic Pressure (CASP) seems to provide more accurate information about the presence of arteriosclerosis and cardiovascular disease-associated end organ damage and thereby the cardiovascular risk profile than brachial BP [13]. However, 24h CASP has not been evaluated in patients with mildly reduced renal function and OSA. Furthermore, non-dipping using $24 \mathrm{~h}$ CASP measurements have not been compared with non-dipping using brachial $24 \mathrm{~h}$ measurements.

We hypothesized that reduced nocturnal BP decrease was associated with estimated Glomerular Filtration Rate (eGFR) and OSA, and that abnormal plasma levels of vasoactive hormones or abnormal renal sodium handling were involved as pathophysiological factors in non-dipping, and that p-Syndecan-1 was a marker of enforced shedding from glycocalyx related to non-dipping. Moreover, we hypothesised that nocturnal dipping status differed if evaluated on brachial or on central measurements.

Thus, in hypertensive patients with mildly reduced renal function (i.e. eGFR $60-89 \mathrm{~mL} / \mathrm{min} / 1.73 \mathrm{~m} 2$ ) and healthy controls, we measured 1. Brachial and central 24h BP, 2. Presence of OSA (Cardio respiratory monitoring), 3. Renal function (as eGFR), 4. P-Syndecan-1, 5. Vasoactive hormones in plasma; renin (PRC), angiotensinII (p-AngII), aldosterone (p-Aldo), vasopressin (p-AVP), and brain natriuretic peptide (p-BNP), and 6. Urinary excretion of sodium (u-Na), aquaporin 2 (u-AQP2), and a component of the epithelial sodium channel (u-ENaC $\gamma$ ).

\section{MATERIALS AND METHODS}

\subsection{Design}

The trial was conducted as a case-control study comparing patients diagnosed with hypertension and eGFR 60-89 $\mathrm{mL} / \mathrm{min} / 1.73 \mathrm{~m}^{2}$ with healthy controls.

\subsection{Study Setting}

The study took place at the University Clinic in Nephrology and Hypertension, Aarhus University and Holstebro Hospital, and The Sleep Apnoea Clinic, Department of Medicine, Holstebro Hospital. The recruitment period was from January 2014 until January 2016.

\subsection{Participants}

All participants were recruited from a population study in Holstebro County $[14,15]$.

Patients were invited, if they had been diagnosed with hypertension in the population study conducted from March 2011 to September 2014. They Hypertension was diagnosed if daytime BP from 24h ambulatory BP measurements $(\mathrm{ABPM}) \geq 135 / 85 \mathrm{mmHg}$. Inclusion criteria were men and women, age 55 to 70 , eGFR 60 to $89 \mathrm{~mL} / \mathrm{min} / 1.73 \mathrm{~m}{ }^{2}$ at sampling time, brachial $24 \mathrm{~h} \mathrm{BP} \geq 130 / 80 \mathrm{mmHg}$ or normal brachial $24 \mathrm{~h}$ BP during antihypertensive treatment. Exclusion criteria were unwillingness to participate, diagnosed OSA prior to participation, increased plasma 
metanephrine, malignant disease, alcohol abuse (more than 21 drinks per week for males and 14 for females), drug abuse, atrial fibrillation or heart failure, liver disease (alanine-aminotransferase $>200$ units/L), severe chronic obstructive lung disease (forced expiratory volume in 1 second $\left(\mathrm{FEV}_{1}\right)$ less than $50 \%$ of expected), and difference in $\mathrm{BP}$ between right and left arm above 10/10 mmHg. Withdrawal criteria were development of exclusion criteria, lack of completion of participation, and lack of compliance.

Controls were invited, if they had normal home BP in the population study. Inclusion criteria were both males and females aged 55-70 years and BMI between 18,5-30 kg/m². Exclusion criteria were the same as for patients and additionally: $24 \mathrm{~h} \mathrm{BP} \geq 130 / 80 \mathrm{mmHg}$, clinical signs or history of diseases in heart, lungs, kidneys, liver, or endocrine organs, smoking or medical treatment. Withdrawal criteria were the same as for patients.

\subsection{Number of Subjects}

Using a significance level of $5 \%$ and a power of $90 \%$, it was calculated that the number of subjects in each group should be 54, when the minimal relevant difference in relative nocturnal BP decrease on $24 \mathrm{~h}$ BP from day to night time was $5 \%$ with SD $8 \%$.

\subsection{Ethics}

This study was approved by the Regional Committees on Health Research Ethics, Denmark (j. no M-2013-303-13) and by the Danish Data Protection Agency (j.no.: 1-16-02-458-13). The study was carried out in accordance with the Helsinki Declaration. All study subjects received oral and written information about the project, and they provided informed written consent prior to study enrolment.

\subsection{Effect Variables}

Primary effect variable was difference between patients and controls in relative brachial nocturnal systolic BP decrease.

Secondary effect variables were brachial $24 \mathrm{~h}$ BP, central 24h BP, PRC, p-AngII, p-Aldo, p-AVP, P-BNP,psyndecan-1, u-AQP2,u-ENaCy, and $24 \mathrm{~h}$ u-Na.

\subsection{Blood Pressure}

Brachial 24h ambulatory BP measurement (ABPM) was measured using an oscillometric device, A \& D TM-2430 (A\&D Company Limited, Tokyo, Japan) with a cuff placed on the right upper arm. The upper arms circumference was measured, and an appropriate size cuff was applied.

Twenty-four hour ambulatory CASP was measured by applanation tonometry using a device, BPro (HealthSTATS, Singapore), placed on the radial artery of the left wrist. The BPro device is using radial applanation tonometry for BP measuring; Central BP is derived by applying n-point moving average method and has previously been validated [16, 17]. The BPro device was calibrated with a mean of the last three of four consecutive BP measured on the left arm with the A\&D device, later used for brachial ABPM on the same subject.

BP was measured every 15 min during daytime and every 30 min overnight by the A\&D device and every 15 min by the BPro device. ABPM for both devices were considered adequate if there were 14 or more daytime recordings (fixed daytime setting at $6.01 \mathrm{am}$ to $10.59 \mathrm{pm}$ ) and seven or more night time recordings (fixed night time setting at 11 pm to $6 \mathrm{am})$.

Bilateral brachial office BP was recorded using a semiautomatic oscillometric device, Omron 705IT (Omron Matsusaka Co, Ltd., Matsusaka City, Japan) with the subject sitting in upright position after minimum ten minutes rest.

Hypertension was defined as brachial $24 \mathrm{~h} \mathrm{BP}>130 \mathrm{mmHg}$ systolic and/or $>80 \mathrm{mmHg}$ diastolic, and non-dipping was defined as relative nocturnal systolic BP decrease $\leq 10 \%$, both according to most recent guidelines from European Society of Hypertension/ European Society of Cardiology [1].

\subsection{Sleep Apnoea}

An ambulatory cardio respiratory monitoring was performed using Embletta Gold (Natus Medical Incorporated, CA, USA). Data were analysed and stored in RemLogic-E Software. More information of the procedure for analysing the cardio respiratory monitoring are given in Supplemental materials. 
Apnoea Hypopnoea Index (AHI) was defined as the sum of apnoeas and hypopneas per hour of registered sleep. Oxygen desaturation index (ODI) was defined as oxygen desaturation events pr. hour of sleep. OSA was defined as $\mathrm{AHI} \geq 5$, moderate to severe $\mathrm{AHI} \geq 15$. OSA definitions are according to the recommendations from The American Academy of Sleep Medicine [18].

\subsection{Experimental Procedure}

Procedures were identical for both patients and controls. All eligible participants were invited by letter, and if necessary, a reminder was sent. Appointment for further information about participation in the study was arranged by telephone or email. Prior to the first meeting, study information was sent. At the first meeting, the participant received oral information about the study. After written consent, further participation was planned. Medical history, use of medication, smoking habits, and alcohol intake were obtained by a questionnaire, and the subjects subjective sleepiness was measured by Epworth Sleepiness Scale (ESS) [19].

Bilateral office BP was measured after a minimum of ten minutes rest. Blood samples were drawn after 20 minutes of rest in supine position. Brachial and central 24h ABPM was performed simultaneously in the following day. The participants were instructed in $24 \mathrm{~h}$ urine collection, and returned it within 4 hours after completion.

Participants were instructed in applying the ambulatory cardio respiratory monitoring a night independent of the ABPM. The participants completed their participation within one month from the time when written consent was provided.

\subsection{Biochemical Analyses}

Blood samples were centrifuged for $10 \mathrm{~min}$ at $2200 \mathrm{x} g$ at $4{ }^{\circ} \mathrm{C}$. Plasma samples were kept frozen at $-20^{\circ} \mathrm{C}(\mathrm{p}$ AngII) and $-80^{\circ} \mathrm{C}$ (PRC, p-Aldo, and p-AVP) until assayed. Urine samples were kept frozen at $-20^{\circ} \mathrm{C}$ until assayed.

Details of the methods used for analyses of p-AngII, PCR, p-BNP, p-Aldo, and p-AVP are provided in the Supplemental material.

P-syndecan-1 was analysed on EDTA-plasma using a human ELISA kit from Abcam plc, Cambridge, United Kingdom. Minimal detection level was $4.94 \mathrm{ng} / \mathrm{mL}$. The coefficients of variations were $10.2 \%$ (inter-assay) and $6.2 \%$ (intra-assay).

P-creatinine, p-HbA1c, p-cholesterols, p-sodium, and urinary concentrations of albumin, creatinine and sodium were measured by routine methods at the Department of Clinical Biochemistry, Holstebro Hospital, Denmark. eGFR was calculated using the MDRD equation.

\subsection{Statistical Methods}

Statistical analyses were performed using IBM SPSS statistics version 22.0 (IBM Corp.; Armonk, NY, United States). All data were tested for normality and variance equality. The statistical level of significance was $p<0.05$ in all analyses. Unless otherwise stated, normally distributed continuous variables were reported as means with SD, continuous non-normally distributed variables were reported as medians with interquartile range [25-75\%]. Unpaired or paired t-test was used for parametric continuous variables. Mann Whitney's test or Wilcoxon's signed rank test was used for non-parametric continuous data. Categorical variables were reported as percentages with number. Chi-square test or Fishers exact test was used to test for association between two categorical variables depending on minimum expected cell count. Correlation analyses were performed with Pearson's test or Spearman Rho test on normally distributed or non-normally distributed continuous variables, respectively.

In a linear regression model, relative brachial nocturnal systolic BP decrease or relative nocturnal CASP decrease were used as dependent variable. Independent variables are mentioned in the specific analyses. The regression analyses were made separately for patients and controls.

\section{RESULTS}

\subsection{Demographics}

Seventy-five patients and 56 controls completed all examinations and were included for analyses (Supplemental material, Fig. S1). Table 1 show clinical and laboratory data; Patients were older, had lower eGFR, and higher BMI. 
Total cholesterol and HDL levels were lower and triglyceride level higher in the hypertensive patients. Among the patients, $77 \%(\mathrm{n}=58)$ received antihypertensive treatment, $60 \%(\mathrm{n}=45)$ received ace-inhibitors or angiotensin II antagonists. Antihypertensive medication was administered only in the morning by 49 patients, only in the evening by one patient or both morning and evening by eight patients. The mean number of BP lowering drugs was 1.4 (95\%CI 1 . $2 ; 1.7)$, the mean period of antihypertensive treatment was 3.4 years $(95 \%$ CI $2.2 ; 4.5), 3 \%(n=2)$ were treated for diabetes, and $20 \%(\mathrm{n}=15)$ were treated with statins.

Table 1. Clinical and laboratory data of hypertensive patients and healthy controls.

\begin{tabular}{|c|c|c|c|}
\hline Characteristics & Controls $(n=56)$ & Patients $(n=75)$ & $p$ \\
\hline Gender, male, $\%(\mathrm{n})$ & $46(26)$ & $55(41)$ & 0.35 \\
\hline Age, years & $62(3)$ & $63(3)$ & 0.022 \\
\hline Office BP, systolic, $\mathrm{mmHg}$ & $130(12)$ & $144(14)$ & $<0.0001$ \\
\hline Body mass index, $\mathrm{kg} / \mathrm{m}^{2}$ & $24(3)$ & $27(5)$ & $<0.0001$ \\
\hline $\mathrm{eGFR}, \mathrm{mL} / \mathrm{min} / 1.73 \mathrm{~m}^{2}$ & $86(12)$ & $80(11)$ & 0.004 \\
\hline Haemoglobin A1c, mmol/mol & $37(3)$ & $37(5)$ & 0.26 \\
\hline Total cholesterol, $\mathrm{mmol} / \mathrm{L}$ & $5.5(0.8)$ & $5.0(1.0)$ & 0.009 \\
\hline HDL cholesterol, $\mathrm{mmol} / \mathrm{L}$ & $1.6(0.4)$ & $1.4(0.4)$ & 0.003 \\
\hline Triglyceride, $\mathrm{mmol} / \mathrm{L}$ & $1.1(0.6)$ & $1.5(0.9)$ & 0.002 \\
\hline Present or former smokers, $\%(n)^{a, b}$ & $48(27)$ & $55(41)$ & 0.47 \\
\hline Weekly alcohol intake, units ${ }^{a, c}$ & $5[2,7]$ & $4[2,10]$ & 0.84 \\
\hline
\end{tabular}

Abbreviations: BP: blood pressure, eGFR: estimated glomerular filtration rate, HDL: high density lipoprotein. Data are presented as mean (SD) except for gender and present smokers, which are presented as percentage (number) and weekly alcohol intake, which is presented as median $[25 ; 75$ percentiles]. ${ }^{a}$ Self-reported data. ${ }^{b}$ None of the controls were present smokers. ${ }^{\mathrm{c}} 1 \mathrm{unit}=12 \mathrm{~g}$ alcohol. Statistics were performed using unpaired t-test, Mann Whitney's test, or chi-square test.

Office systolic/diastolic BP difference between right and left arm was $-0.5 \pm 5.0 /-0.4 \pm 3.6 \mathrm{mmHg}(p=0.41 / p=0.31)$ in patients and $-1.0 \pm 5.3 /-1.2 \pm 3.9 \mathrm{mmHg}(p=0.18 / p=0.022)$ in the controls.

\subsection{Brachial Ambulatory Blood Pressure Measurement}

Table 2 shows results from brachial ABPM. As expected, patients had higher BP than controls. The mean absolute nocturnal systolic BP (SBP) decrease did not differ significantly between hypertensive patients and controls, whereas the corresponding relative decrease was significantly lower in hypertensive patients than in controls. There was no difference in absolute or relative nocturnal SBP decrease between patients receiving antihypertensive treatment only in the morning and patients receiving antihypertensive treatment in the evening ( $p=0.71$ and $p=0.94$, respectively). Among patients, 12 (19\%) had normal ABPM during antihypertensive treatment.

\subsection{Ambulatory CASP Measurement}

Eighteen participant's central ABPM were excluded from analyses due to outlying ( $>$ 3SD from mean, one patient), too few measurements during day (six patients and two controls) or night period (four patients and five controls). Thus, the CASP analysis included 64 patients and 49 controls. Patients had higher CASP than controls. The mean absolute nocturnal CASP decrease did not differ significantly between hypertensive patients and controls, whereas the corresponding relative decrease was significantly lower in hypertensive patients.

Table 2. Brachial and central blood pressure from 24h ABPM in hypertensive patients and healthy controls.

\begin{tabular}{|c|c|c|c|c|c|c|}
\hline & \multicolumn{3}{|c|}{ Brachial BP } & \multicolumn{3}{|c|}{ CASP } \\
\hline & Controls $(n=56)$ & Patients $(n=75)$ & $\begin{array}{c}p \\
\text { Brachial }\end{array}$ & Controls $(n=49)$ & Patients $(n=64)$ & $\begin{array}{c}p \\
\text { CASP }\end{array}$ \\
\hline 24h systolic $\mathrm{BP}, \mathrm{mmHg}$ & $120(8)$ & $139(11)$ & $<0.0001$ & $109(16)^{* *}$ & $124(14)^{* *}$ & $<0.0001$ \\
\hline 24h diastolic $\mathrm{BP}, \mathrm{mmHg}$ & $73(4)$ & $82(6)$ & $<0.0001$ & & & \\
\hline $24 \mathrm{~h}$ heart rate, beat $/ \mathrm{min}$ & $67(9)$ & $69(10)$ & 0.19 & & & \\
\hline Daytime systolic BP, $\mathrm{mmHg}$ & $124(9)$ & $141(11)$ & $<0.0001$ & $113(15)^{* *}$ & $127(15)^{* *}$ & $<0.0001$ \\
\hline Daytime diastolic BP, $\mathrm{mmHg}$ & $75(5)$ & $84(7)$ & $<0.0001$ & & & \\
\hline Night-time systolic $\mathrm{BP}, \mathrm{mmHg}$ & $103(8)$ & $123(15)$ & $<0.0001$ & $102(15)$ & $117(15)^{*}$ & $<0.0001$ \\
\hline Night-time diastolic $\mathrm{BP}, \mathrm{mmHg}$ & $63(5)$ & $73(9)$ & $<0.0001$ & & & \\
\hline
\end{tabular}


(Table 2) contd.....

\begin{tabular}{|c|c|c|c|c|c|c|}
\hline & \multicolumn{3}{|c|}{ Brachial BP } & \multicolumn{3}{|c|}{ CASP } \\
\hline & Controls $(n=56)$ & Patients $(n=75)$ & $\begin{array}{c}p \\
\text { Brachial }\end{array}$ & Controls $(n=49)$ & Patients $(n=64)$ & $\begin{array}{c}p \\
\text { CASP }\end{array}$ \\
\hline Relative nocturnal SBP decrease, $\%$ & $17(7)$ & $13(7)$ & 0.002 & $10(5) * *$ & $7(6) * *$ & 0.019 \\
\hline $\begin{array}{l}\text { Absolute nocturnal SBP decrease, } \\
\mathrm{mmHg}\end{array}$ & $21(9)$ & $18(10)$ & 0.09 & $11(6)^{* *}$ & $10(8) * *$ & 0.24 \\
\hline Non-dippers, $\%(n)^{a}$ & $18(10)$ & $32(24)$ & 0.07 & $50(26)^{*}$ & $69(46)^{* *}$ & 0.032 \\
\hline
\end{tabular}

Abbreviations: ABPM: Ambulatory Blood Pressure Monitoring, BP: Blood Pressure, CASP: Central Aortic Systolic Pressure, SBP: Systolic Blood Pressure. Data are presented as mean (SD) unless otherwise stated. ${ }^{a}$ Percentage (number) of subjects with nocturnal systolic BP decrease $<10 \%$ of daytime. * Compared to brachial BP within the same group, $p<0.05$. ${ }^{*}$ Compared to brachial BP within the same group, $p<0.0001$. Statistics were performed using unpaired t-test or chi-square test between groups, and paired t-test or McNemars test within groups.

$24 \mathrm{~h}$, day and night-time CASP, absolute and relative nocturnal CASP decrease were lower than corresponding brachial values in both patients and controls, except for night-time CASP in controls.

\subsection{Predictive Value of CASP Non-Dipping}

According to guidelines from European Society of Hypertension, non-dipping is defined as nocturnal systolic BP decrease $<10 \%$ of daytime value using brachial ABPM. Table 3 shows the predictive values of using CASP measurements to diagnose non-dipping correctly with ABPM as reference using a $10 \%$ dipping limit. A $5 \%$ and $10 \%$ CASP dipping limit was used. The predictive value of diagnosing non-dipping was higher in patients than in controls; $10 \%$ nocturnal CASP decrease limit predicted $38 \%$ and $17 \%$ of brachial non-dippers in patients and controls, respectively, whereas, $5 \%$ nocturnal CASP decrease limit predicted $25 \%$ and $14 \%$ of brachial non-dippers in patients and controls, respectively.

Table 3. The predictive value of using $24 \mathrm{~h}$ CASP to diagnose non-dipping with $24 \mathrm{~h}$ brachial dipping as reference in hypertensive patients and healthy controls.

\begin{tabular}{|c|c|c|}
\hline & Controls (n=49) & Patients (n=64) \\
\hline & \multicolumn{2}{|c|}{ Predictive Value of Non-Dipping } \\
\hline $10 \%$ nocturnal CASP decrease & $17 \%$ & $38 \%$ \\
\hline $5 \%$ nocturnal CASP decrease & $14 \%$ & $25 \%$ \\
\hline
\end{tabular}

Abbreviation: CASP: Central Aortic Systolic Pressure.

Calculations: Predictive value of non-dipping $=($ CASP and brachial non-dippers $) /(\text { all CASP non-dippers })^{*} 100$.

\subsection{Cardio Respiratory Monitoring}

Results from cardio respiratory monitoring and Epworth Sleepiness Scale (ESS) are seen in Table 4. Patients had higher ODI and longer snoring time than controls. Moderate-to-severe OSA was more frequently diagnosed in patients than in controls $(17 \% v s .2 \%, p=0.004)$, whereas the frequency of all-degree OSA did not differ significantly ( $24 \% v s$. $14 \%, p=0.38)$. There was no significant difference in AHI, mean oxygen saturation $\left(\mathrm{SaO}_{2}\right)$, or ESS between patients and controls. Mean cardio respiratory monitoring time was shorter in patients than controls (363 (95\%CI 344;381) min $v s$. $401(95 \%$ CI $385 ; 417)$ min, $p=0.003)$.

Table 4. Epworth sleepiness scale and cardio respiratory monitoring in hypertensive patients and healthy controls.

\begin{tabular}{|c|c|c|c|}
\hline & Controls $(n=56)$ & Patients $(n=75)$ & $p$ \\
\hline ESS, $0-24^{\text {a }}$ & $4[2,7]$ & $5[3,8]$ & 0.27 \\
\hline AHI, events/hour & $1.8[2.2 ; 4.3]$ & $1.7[0.7 ; 6.6]$ & 0.44 \\
\hline ODI, events/hour & $1.7[0.7 ; 4.4]$ & $3.1[1.1 ; 7.6]$ & 0.014 \\
\hline Snoring, $\%$ of sleep time & $0.3[0.0 ; 2.0]$ & $0.8[0.0 ; 8.3]$ & 0.016 \\
\hline Mean $\mathrm{SaO}_{2}, \%$ & $94.5(1.3)$ & $93.8(1.9)$ & 0.06 \\
\hline $\mathrm{AHI} \geq 5, \%(\mathrm{n})^{\mathrm{b}}$ & $25(14)$ & $32(24)$ & 0.38 \\
\hline $\mathrm{AHI} \geq 15, \%(\mathrm{n})^{\mathrm{c}}$ & $2(1)$ & $17(13)$ & 0.004 \\
\hline
\end{tabular}

Abbreviation: AHI: Apnoea hypopnea index, ESS: Epworth Sleepiness Scale, ODI: Oxygen desaturation index, $\mathrm{SaO}_{2}$ : Mean Oxygen Saturation. ESS, $\mathrm{AHI}$, ODI and snoring are presented as median with interquartile range [25; 75\%]. Mean $\mathrm{SaO}_{2}$ is presented as mean (SD). AHI $\geq 5$ and $\geq 15$ are presented as percentage (number). ${ }^{\mathrm{a}}$ From Epworth Sleepiness Scale, self-reported data. ${ }^{\mathrm{b}}$ All-degree OSA, ${ }^{\mathrm{c}}$ Moderate-to-severe OSA Statistics were performed using unpaired t-test or Mann-Whitney's test, chi-squared test or Fishers exact test. 


\subsection{Plasma Levels of Syndecan-1}

As seen in Table 5, patients had significantly higher levels of p-syndecan-1 than controls (14.0 [8. 2; 20.8] vs. 9.3 $[7.9 ; 17.9] \mathrm{ng} / \mathrm{mL}, p=0.039)$. There was no difference between non-dippers and dippers within each group. Psyndecan-1 was not correlated to nocturnal BP decrease, occurrence of OSA, BMI, or eGFR in either group (data not shown).

Table 5. Plasma syndecan-1, plasma hormone levels and urine analyses in hypertensive patients and healthy controls.

\begin{tabular}{|c|c|c|c|c|c|c|c|}
\hline \multirow[b]{2}{*}{ Plasma } & \multicolumn{3}{|c|}{ Controls } & \multicolumn{3}{|c|}{ Patients } & \multirow[b]{2}{*}{$p$-total } \\
\hline & Total $(\mathrm{n}=56)$ & Dipper $(n=46)$ & Non-dipper $(n=10)$ & Total $(n=75)$ & Dipper $(n=51)$ & Non-dipper $(n=24)$ & \\
\hline Syndecan-1, ng/mL & $9.3[7.9 ; 17.9]$ & $8.8[7.9 ; 16.5]$ & $10.6[7.9 ; 23.4]$ & $14.0[8.2 ; 20.8]$ & $14.2[8.5 ; 19.2]$ & $11.3[7.9 ; 26.9]$ & 0.039 \\
\hline Aldo, $\mathrm{pmol} / \mathrm{L}$ & $88.1(49.1)$ & $88.5(47.7)$ & $86.3(57.5)$ & $98.3(57.0)$ & $97.3(52.7)$ & $100.6(66.5)$ & 0.28 \\
\hline AngII, ng/L & $8.0[5.3 ; 10.8]$ & $8.0[6.0 ; 10.3]$ & $7.0[4.8 ; 12.3]$ & $9.0[5.0 ; 18.0]$ & $10.0[5.0 ; 22,0]$ & $9.0[5.0 ; 17.0]$ & 0.17 \\
\hline PRC, ng/L & $6.1[4.4 ; 8.8]$ & $6.4[4.7 ; 8.9]$ & $4.5[4.1 ; 7.8]$ & $12.7[5.6 ; 31.9]$ & $15.2[6.0 ; 43.0]$ & $11.8[4.9 ; 19.1]$ & $<0.0001$ \\
\hline $\mathrm{AVP}, \mathrm{ng} / \mathrm{L}$ & $0.4[0.3 ; 0.5]$ & $0.4[0.3 ; 0.5]$ & $0.4[0.3 ; 0.5]$ & $0.4[0.3 ; 0.5]$ & $0.4[0.2 ; 0.5]$ & $0.4[0.3 ; 0.4]$ & 0.55 \\
\hline $\mathrm{BNP}, \mathrm{pmol} / \mathrm{L}$ & $4.4[2.9 ; 7.7]$ & $4.4[2.9 ; 7.7]$ & $4.4[2.9 ; 8.7]$ & $4.0[2.9 ; 6.8]$ & $3.66[2.88 ; 7.38]$ & $4.68[3.62 ; 7.38]$ & 0.73 \\
\hline \multicolumn{8}{|l|}{ Urine } \\
\hline $24 \mathrm{~h} \mathrm{u}-\mathrm{Na}, \mathrm{mmol} / 24 \mathrm{~h}$ & $144(47)$ & $143(47)$ & $152(49)$ & $155(53)$ & $159(57)$ & $147(43)$ & 0.23 \\
\hline $\mathrm{AQP} 2, \mathrm{ng} / \mathrm{min}$ & $0.94[0.78 ; 1.34]$ & $0.92[0.78 ; 1.34]$ & $1.11[0.70 ; 1.55]$ & $1.10[0.87 ; 1.40]$ & $1.23[0.86 ; 1.44]$ & $1.05[0.93 ; 1.29]$ & 0.14 \\
\hline $\mathrm{ENaCy}, \mathrm{ng} / \mathrm{min}$ & $0.82[0.67 ; 1.21]$ & $0.80[0.65 ; 1.11]$ & $1.02[0.67 ; 1.37]$ & $1.02[0.81 ; 1.30]$ & $1.05[0.81 ; 1.31]$ & $1.01[0.78 ; 1.20]$ & 0.016 \\
\hline
\end{tabular}

Abbreviations: Aldo: Aldosterone, AQP2: urine excretion of aquaporin-2, AngII: angiotensin II, AVP: arginine vasopressin, BNP: brain natriuretic peptide, ENaCy: urine excretion of epithelial sodium channel $\gamma$, 24h u-Na: 24-h urinary sodium excretion, PRC: plasma renin concentration. Aldo and 24h u-Na are presented as mean (SD). The remaining are presented as median with interquartile range [25;75\%]. Unpaired t-test or Mann Whitney's test was made between cases and controls; the $p$-value of this test is shown in the table. Unpaired t-test or Mann Whitney's test was also performed between dippers and non-dippers within the case and within the control group; this showed no significance.

\subsection{Plasma Levels of Hormones}

Plasma levels of vasoactive hormones are shown in Table 5. Patients had significantly higher mean PRC than controls $(12.7$ [5.6; 31.9] vs. 6.1 [4.4; 8.8] ng/L, $p<0.0001)$. There were no differences between patients and controls with regard to the other hormone levels (Aldo, AngII, AVP, and BNP), and no significant differences between dippers and non-dippers within each group. Plasma levels of hormones were not correlated to nocturnal brachial BP decrease or CASP decrease in either patients or controls (data not shown).

\subsection{Sodium in Urine and Urinary Excretion of $\mathrm{AQP} 2$ and $\mathrm{ENaCy}$}

Results from urine analyses are displayed in Table 5. Urinary excretion rate of $\mathrm{ENaC \gamma}$ was higher in patients, also when excluding patients receiving thiazide from the analyses.

In the patient group, univariate correlation analyses showed correlation between $24 \mathrm{~h} \mathrm{u}-\mathrm{Na}$ and the following: $24 \mathrm{~h}$ brachial BP $(r=0.306, p=0.008)$ and daytime brachial BP $(r=0.320, p=0.005)$. None of these correlations were found in controls.

\subsection{Associations of Nocturnal Blood Pressure Decrease}

Table 6 lists results from the regression analysis with brachial nocturnal BP decrease as dependent variable and presence of OSA, eGFR, BMI, and $24 \mathrm{~h} \mathrm{u}-\mathrm{Na}$ as independent variables for patients and controls. This regression model did not predict association of OSA, eGFR, BMI, or $24 \mathrm{~h}$ urine sodium on nocturnal BP decrease for either patients or controls.

In a regression analysis with brachial nocturnal BP decrease as dependent variable and p-syndecan-1, presence of OSA, eGFR, and $24 \mathrm{~h} \mathrm{u}-\mathrm{Na}$ as independent variables, none of the partial regression coefficients reached statistical significance in either patients or controls (data not shown). Another regression analysis using the same dependent variable and logarithmic transformed plasma hormone levels (p-AngII, PRC, p-Aldo, p-BNP, p-AVP) as independent variables showed no significance of the partial regression coefficients (data not shown). 
Table 6. Regression analysis with relative brachial nocturnal blood pressure decrease as dependent variable in hypertensive patients and healthy controls.

\begin{tabular}{|c|c|c|c|c|c|c|}
\hline Patients & \multicolumn{3}{|c|}{ B $(95 \%$ CI $)$} & SE B & $\beta$ & $p$ \\
\hline Constant & 17.07 & $(1.05$ & 33.09) & 8.03 & & 0.04 \\
\hline eGFR, $\mathrm{mL} / \mathrm{min} / 1.73 \mathrm{~m}^{2}$ & -0.01 & $(-0.17$ & $0.15)$ & 0.08 & -.01 & 0.93 \\
\hline Presence of OSA & -1.48 & $(-5.66$ & $2.69)$ & 2.09 & -.10 & 0.48 \\
\hline Body mass index, $\mathrm{kg} / \mathrm{m}^{2}$ & -0.24 & $(-0.66$ & $0.19)$ & 0.21 & -.15 & 0.27 \\
\hline $24 \mathrm{~h} \mathrm{u}-\mathrm{Na}, \mathrm{mmol} / 24$ hour & 0.02 & $(-0.02$ & $0.06)$ & 0.02 & .14 & 0.28 \\
\hline \multicolumn{7}{|c|}{ R Square for this model: $0.05, p=0.51$} \\
\hline Controls & \multicolumn{3}{|c|}{ B (95\%CI) } & SE B & $\boldsymbol{\beta}$ & $p$ \\
\hline Constant & 18.19 & $(-6.64$ & $43.01)$ & 12.36 & & 0.15 \\
\hline eGFR, $\mathrm{mL} / \mathrm{min} / / 1.73 \mathrm{~m}^{2}$ & 0.00 & $(-0.18$ & $0.18)$ & 0.09 & .00 & 0.99 \\
\hline Presence of OSA & 2.43 & $(-1.99$ & $6.86)$ & 2.20 & .16 & 0.27 \\
\hline Body mass index, $\mathrm{kg} / \mathrm{m}^{2}$ & -0.14 & $(-1.08$ & $0.81)$ & 0.47 & -.05 & 0.77 \\
\hline $24 \mathrm{~h} \mathrm{u}-\mathrm{Na}, \mathrm{mmol} / 24$ hour & 0.01 & $(-0.05$ & $0.06)$ & 0.03 & .04 & 0.82 \\
\hline
\end{tabular}

Abbreviation: eGFR: estimated Glomerular Filtration Rate, OSA: Obstructive Sleep Apnoea, 24h u-Na: 24h urinary sodium excretion. Statistics were performed using a linear regression model. Dependent variable was relative nocturnal brachial systolic BP decrease. Independent variables were eGFR, presence of OSA (AHI > 5), BMI, and 24h U-Na.

\section{DISCUSSION}

The main finding in this study was lower nocturnal BP decrease using both brachial and central ABPM in hypertensive patients with mildly reduced renal function compared with healthy controls. Moderate-to-severe OSA occurred more frequently among hypertensive patients. The nocturnal BP decrease was not associated with the presence of OSA or the level of eGFR using either brachial or central BP measurements.

As expected, we measured a relatively smaller reduction in nocturnal BP in hypertensive patients compared with controls using brachial ABPM. In addition, we measured BP using CASP during the same 24 hours as brachial ABPM, and the difference in nocturnal BP decrease was the same between the two groups as for measurements using brachial ABPM. However, the effect of OSA, a highly prevalent co morbidity in hypertensive patients [20], on nocturnal BP decrease in has not yet been described. In our study, nocturnal BP decrease was not associated with the presence of OSA, using either univariate correlation analysis or multiple regression analysis with inclusion of eGFR, BMI, and 24 hours urinary sodium excretion as additional independent variables. In other studies comprising primarily men referred to a sleep clinic or obese OSA subjects, OSA was related to nocturnal BP decrease and non-dipping [5, 21]. However, patients from these studies were not comparable to the present study. We included a population based on hypertension, whereas the prior studies included patients based on diagnosis of OSA [5] or referral to examination for OSA [21]. In addition to this, patients from the previous studies had higher BMI and were younger than our patients. Renal function and diabetes status were not taken into consideration in the previous studies. We aimed to clarify whether occurrence of OSA was associated with nocturnal BP decrease in a hypertensive population selected based on BP criteria, not OSA susceptibility. We could not prove the existence of this association. According to our information, this is new knowledge regarding the relationship between OSA and nocturnal BP decrease in hypertensive patients.

In the present study, we diagnosed moderate-to-severe OSA $(\mathrm{AHI} \geq 15)$ in $17 \%$ of mildly overweight hypertensive patients in contrast to only $2 \%$ of healthy controls. The prevalence of moderate-to-severe OSA in our the hypertensive population was the same as measured in another study of hypertensive patients using systematic screening [22], but higher than reported in studies of hypertensive patients using diagnoses directly from a coding systems for OSA diagnosis [10, 23]. We did not invite subjects with diagnosed OSA prior to participation. Thus, differences in diagnostic criteria may explain the discrepancy, as well as under diagnosing of OSA in the general population. The occurrence of non-dipping have been shown to be increased in patients with considerable reduction in renal function $[8,10]$, but it is unknown at which stage non-dipping begins in patients with deteriorating renal function. In the present study, hypertensive patients were sampled with mildly reduced renal function i.e. eGFR $60-89 \mathrm{~mL} / \mathrm{min} / 1.73 \mathrm{~m}^{2}$. We found no correlation between the nocturnal BP decrease and eGFR using either univariate correlation analysis or multiple regression analysis with inclusion of OSA, BMI, and 24h urinary sodium excretion as additional independent variables. The results were the same independent of analysis based on $24 \mathrm{~h}$ brachial or central BP. Reduced nocturnal BP decrease 
is well-documented in the literature in patients with severely reduced renal function. However, in patients with mildly reduced renal function, i.e. eGFR between 60 and $89 \mathrm{~mL} / \mathrm{min} / 1.73 \mathrm{~m}^{2}$, the present study showed no significant association between nocturnal BP decrease and eGFR. We only included a narrow eGFR span in this study; the results might have been different if patients with renal function in a wider span had been included.

Our analysis showed that BMI was not related to nocturnal BP decrease in either patients or controls. These findings are in agreement with a previous study [24]. However, from other studies BMI have been reported markedly higher for OSA subjects [25]. In the present study, BMI diverged $3 \mathrm{~kg} / \mathrm{m}^{2}$ between patients and controls. Thus, most likely, a difference in BMI can not explain the high frequency of OSA in our mildly-overweight patients.

When we compared BP data between brachial 24h ABPM and 24h CASP, BP was lower using CASP, i.e. during the entire $24 \mathrm{~h}$ period and during both day- and night-time, the latter except for controls. This was an expected finding [26]. We estimated the positive predictive value of $10 \%$ and $5 \%$ CASP dipping to diagnose non-dipping using brachial $10 \%$ dipping limit as diagnostic standard. The purpose was to examine if an optimal limit could be established, at which patients were correctly classified as non-dippers. However, we found that a significant number of non-dippers in both groups would be misclassified as dippers regardless of CASP dipping limit used. The CAFÉ study found different central and brachial BP response to antihypertensive treatment, which was hypothesised to explain the difference in cardiovascular outcome despite similar brachial BP response [27]. According to our study, the occurrence of nondipping deviates much depending on the evaluation methods, i.e. between $24 \mathrm{~h}$ brachial and CASP ABPM. Further studies are necessary to show which methods are most valuable to predict risk of cardiovascular disease using nondipping as risk marker.

We measured a clearly higher level of p-syndecan-1 in the hypertensive patients than in controls (14.0 vs. 9.3 $\mathrm{ng} / \mathrm{mL})$. No significant difference existed between p-syndecan-1 level in dippers and non-dippers in either patients or controls, and nocturnal BP decrease was not significantly related to p-syndecan-1 either in univariate analysis or in multiple regression analysis where eGFR, OSA, and $24 \mathrm{~h}$ urinary sodium excretion were included as independent variables. Previous studies have demonstrated higher levels of p-syndecan associated to overt heart failure in hypertensive patients and to decreasing renal function in CKD patients [28, 29]. The higher syndecan-1 levels in our group of hypertensive patients indicated that cardiovascular stress was present in these patients even though they only had a low disease burden, i.e. mildly reduced renal function and mild overweight. We aimed to examine the levels of syndecan-1 as a potential marker of damage to the endothelial glycocalyx in relation to non-dipping, but apparently, $\mathrm{p}$ syndecan-1 does not reflect the risk of cardiovascular disease due to a lower reduction in nocturnal BP decrease. It is an interesting and novel finding that damage to the endothelial glycocalyx layer could be revealed in hypertensive patients with mildly reduced renal function. It is not clarified whether the injury of the endothelium is due to hypertension, renal disease, or a combination.

In the present study, we did not measure any difference in plasma level of PRC, p-AngII, p-Aldo, p-AVP, and pBNP between dippers and non-dippers in either hypertensives or controls. No relationship existed between nocturnal BP decrease and either of the hormones in univariate or multivariate analysis. Another study found no difference between dippers and non-dippers with regard to renin and aldosterone [30], whereas Satoh et al. found association of aldosterone-to-renin ratio and non-dipping [31]; the reason for this discrepancy is not known. We measured a higher PRC in patients than controls, presumably, due to treatment with ACE-inhibitors. Our result does not support the view that non-dipping could be attributed to an abnormal plasma level of vasoactive hormones.

We did not demonstrate that sodium balance and renal handling of sodium and water were related to non-dipping, but we measured a higher renal excretion rate of $\mathrm{ENaCy}$ in hypertensive patients compared to controls. Increased urinary excretion of $\mathrm{ENaC} \gamma$ reflects increased sodium transport from the tubular lumen to the intracellular space via the epithelial sodium channels, located in the principal cells in the distal part of the nephron [32 - 35]. Even when excluding patients receiving diuretics, this difference was still significant. In addition, $\mathrm{u}-\mathrm{Na}$, which is a marker of dietary sodium intake, was correlated to BP parameters (24h and daytime BP), but not a determinant for nocturnal BP decrease or nondipping. None of these correlations existed in healthy subjects. The higher excretion rate of ENaCy in the present study suggests altered handling of sodium in the nephron in the hypertensive population. This mechanism might contribute to development and maintenance of high BP by reabsorption of sodium and water enhancing the extracellular volume and amplifying BP.

The study comprised well-matched patient and control groups regarding age and gender, and both hypertensive patients and healthy controls lived in the same area of the country. We measured brachial and central ABPM 
simultaneously. A systematic screening for OSA was done. We included a novel potential marker for vascular damage and several vasoactive hormones in the analyses. It is a weakness of the study that some hypertensive patients received antihypertensive medication, but it was not considered ethically justified to discontinue all antihypertensive agents.

\section{CONCLUSION}

The study showed a lower nocturnal BP decrease using both brachial and central ABPM in hypertensive patients with mildly reduced renal function compared with healthy controls. Moderate-to-severe OSA occurred more frequently among the hypertensives. The decrease in nocturnal BP was not associated with occurrence of OSA or the level of eGFR using either brachial or central ABPM, and was not associated to plasma level of vasoactive hormones. The positive predictive value of non-dipping was poor using CASP with brachial ABPM as reference. Damage to the endothelial glycocalyx layer was revealed in the hypertensive patients with mildly reduced renal function using measurement of syndecan in plasma.

\section{FUNDING}

This research project has received research funding from The Central Denmark Region's Research Foundation for Health Science, The Danish Heart Association, and the Axel Muusfeldt Foundation.

\section{LIST OF ABBREVIATIONS}

$\begin{array}{lll}\text { ABPM } & = & \text { Ambulatory Blood Pressure Measurement } \\ \text { Aldo } & = & \text { Aldosterone } \\ \text { AQP2 } & = & \text { Aquaporin } 2 \\ \text { AngII } & = & \text { Angiotension II } \\ \text { AVP } & = & \text { Arginine Vasopressin } \\ \text { BMI } & = & \text { Body Mass Index } \\ \text { BNP } & = & \text { Brain Natriuretic Peptide } \\ \text { BP } & = & \text { Blood Pressure } \\ \text { CASP } & = & \text { Central Aortic Systolic Pressure } \\ \text { CKD } & = & \text { Chronic Kidney Disease } \\ \text { eGFR } & = & \text { Estimated Glomerular Filtration Rate } \\ \text { ENaC } \gamma & = & \text { Epithelial Sodium Channel } \gamma \\ \text { ESS } & = & \text { Epworth Sleepiness Scale } \\ \text { OSA } & = & \text { obstructive Sleep Apnoea } \\ \text { PRC } & = & \text { Plasma Renin Concentration }\end{array}$

\section{ETHICS APPROVAL AND CONSENT TO PARTICIPATE}

This study was approved by the Regional Committees on Health Research Ethics, Denmark (j. no M-2013-303-13) and by the Danish Data Protection Agency (j. no.: 1-16-02-458-13).

\section{HUMAN AND ANIMAL RIGHTS}

No Animals were used in this research. All human research procedures followed were in accordance with the ethical standards of the committee responsible for human experimentation (institutional and national), and with the Helsinki Declaration of 1975, as revised in 2013.

\section{CONSENT FOR PUBLICATION}

Informed consent have been obtained.

\section{CONFLICT OF INTEREST}

The authors declare no conflict of interest, financial or otherwise. 


\section{ACKNOWLEDGEMENTS}

The authors greatly acknowledge the skillful assistance of the laboratory technicians in the University Clinic of Nephrology and Hypertension: Anne Mette Ravn, Kirsten Nygaard and Henriette Vorup Simonsen.

The authors also greatly acknowledge the skillful assistance from nurses Marianne Kirkegaard and Anja Mailund Mikkelsen in the Sleep Apnoea Clinic, Department of Medicine, Holstebro Hospital, Denmark.

The authors thank the Department of Clinical Biochemistry, Holstebro Hospital for help in BNP and routine analyses.

\section{SUPPLEMENTARY MATERIAL}

Supplementary material is available on the publishers Web site along with the published article.

\section{REFERENCES}

[1] Mancia G, Fagard R, Narkiewicz K, et al. 2013 ESH/ESC Practice guidelines for the management of arterial hypertension. Blood Press 2014; 23(1): 3-16. [http://dx.doi.org/10.3109/08037051.2014.868629] [PMID: 24359485]

[2] Salles GF, Reboldi G, Fagard RH, et al. Prognostic effect of the nocturnal blood pressure fall in hypertensive patients: The ambulatory blood pressure collaboration in patients with hypertension (ABC-H) meta-analysis. Hypertension 2016; 67(4): 693-700. [http://dx.doi.org/10.1161/HYPERTENSIONAHA.115.06981] [PMID: 26902495]

[3] Hansen TW, Li Y, Boggia J, Thijs L, Richart T, Staessen JA. Predictive role of the nighttime blood pressure. Hypertension 2011; 57(1): 3-10. [http://dx.doi.org/10.1161/HYPERTENSIONAHA.109.133900] [PMID: 21079049]

[4] Nicholl DDM, Ahmed SB, Loewen AHS, et al. Declining kidney function increases the prevalence of sleep apnea and nocturnal hypoxia. Chest 2012; 141(6): 1422-30.

[http://dx.doi.org/10.1378/chest.11-1809] [PMID: 22222188]

[5] Nabe B, Lies A, Pankow W, Kohl FV, Lohmann FW. Determinants of circadian blood pressure rhythm and blood pressure variability in obstructive sleep apnoea. J Sleep Res 1995; 4(S1): 97-101. [http://dx.doi.org/10.1111/j.1365-2869.1995.tb00196.x] [PMID: 10607183]

[6] Worsnop CJ, Naughton MT, Barter CE, Morgan TO, Anderson AI, Pierce RJ. The prevalence of obstructive sleep apnea in hypertensives. Am J Respir Crit Care Med 1998; 157(1): 111-5.

[http://dx.doi.org/10.1164/ajrccm.157.1.9609063] [PMID: 9445287]

[7] Lavie P, Yoffe N, Berger I, Peled R. The relationship between the severity of sleep apnea syndrome and 24-h blood pressure values in patients with obstructive sleep apnea. Chest 1993; 103(3): 717-21. [http://dx.doi.org/10.1378/chest.103.3.717] [PMID: 8449057]

[8] Fedecostante M, Spannella F, Cola G, Espinosa E, Dessì-Fulgheri P, Sarzani R. Chronic kidney disease is characterized by “ double trouble” higher pulse pressure plus night-time systolic blood pressure and more severe cardiac damage. PLoS One 2014; 9(1): e86155. [http://dx.doi.org/10.1371/journal.pone.0086155] [PMID: 24465931]

[9] Middeke M, Schrader J. Nocturnal blood pressure in normotensive subjects and those with white coat, primary, and secondary hypertension. BMJ 1994; 308(6929): 630-2.

[http://dx.doi.org/10.1136/bmj.308.6929.630] [PMID: 8148712]

[10] Mojón A, Ayala DE, Piñeiro L, et al. Comparison of ambulatory blood pressure parameters of hypertensive patients with and without chronic kidney disease. Chronobiol Int 2013; 30(1-2): 145-58. [http://dx.doi.org/10.3109/07420528.2012.703083] [PMID: 23181690]

[11] Tarbell JM, Cancel LM. The glycocalyx and its significance in human medicine. J Intern Med 2016; $280(1): 97-113$. [http://dx.doi.org/10.1111/joim.12465] [PMID: 26749537]

[12] Higashi Y, Nakagawa K, Kimura M, et al. Circadian variation of blood pressure and endothelial function in patients with essential hypertension: A comparison of dippers and non-dippers. J Am Coll Cardiol 2002; 40(11): 2039-43. [http://dx.doi.org/10.1016/S0735-1097(02)02535-4] [PMID: 12475467]

[13] Roman MJ, Devereux RB, Kizer JR, et al. Central pressure more strongly relates to vascular disease and outcome than does brachial pressure: The Strong Heart Study. Hypertension 2007; 50(1): 197-203. [http://dx.doi.org/10.1161/HYPERTENSIONAHA.107.089078] [PMID: 17485598]

[14] Hoffmann-Petersen N, Lauritzen T, Bech JN, Pedersen EB. Short-term telemedical home blood pressure monitoring does not improve blood pressure in uncomplicated hypertensive patients. J Hum Hypertens 2017; 31(2): 93-8. [http://dx.doi.org/10.1038/jhh.2016.43] [PMID: 27334521] 
[15] Hoffmann-Petersen N, Lauritzen T, Bech JN, Pedersen EB. High prevalence of hypertension in a danish population telemedical home measurement of blood pressure in citizens aged 55-64 years in holstebro county. Am J Hypertens 2016; 29(4): $439-47$. [http://dx.doi.org/10.1093/ajh/hpv116] [PMID: 26208671]

[16] Williams B, Lacy PS, Yan P, Hwee CN, Liang C, Ting CM. Development and validation of a novel method to derive central aortic systolic pressure from the radial pressure waveform using an n-point moving average method. J Am Coll Cardiol 2011; 57(8): 951-61. [http://dx.doi.org/10.1016/j.jacc.2010.09.054] [PMID: 21329842]

[17] Ott C, Haetinger S, Schneider MP, Pauschinger M, Schmieder RE. Comparison of two noninvasive devices for measurement of central systolic blood pressure with invasive measurement during cardiac catheterization. J Clin Hypertens (Greenwich) 2012; 14(9): 575-9. [http://dx.doi.org/10.1111/j.1751-7176.2012.00682.x] [PMID: 22947354]

[18] Berry RB, Budhiraja R, Gottlieb DJ, et al. Rules for scoring respiratory events in sleep: Update of the 2007 AASM manual for the scoring of sleep and associated events. J Clin Sleep Med 2012; 8(5): 597-619. [PMID: 23066376]

[19] Chiu HY, Chen PY, Chuang LP, et al. Diagnostic accuracy of the berlin questionnaire, STOP-BANG, STOP, and epworth sleepiness scale in detecting obstructive sleep apnea: A bivariate meta-analysis. Sleep Med Rev 2016. [PMID: 27919588]

[20] Nieto FJ, Young TB, Lind BK, et al. Association of sleep-disordered breathing, sleep apnea, and hypertension in a large community-based study. Sleep heart health study. JAMA 2000; 283(14): 1829-36.

[http://dx.doi.org/10.1001/jama.283.14.1829] [PMID: 10770144]

[21] Pankow W, Nabe B, Lies A, et al. Influence of sleep apnea on 24-hour blood pressure. Chest 1997; 112(5): $1253-8$. [http://dx.doi.org/10.1378/chest.112.5.1253] [PMID: 9367465]

[22] Prejbisz A, Florczak E, Pręgowska-Chwała B, et al. Relationship between obstructive sleep apnea and markers of cardiovascular alterations in never-treated hypertensive patients. Hypertens Res 2014; 37(6): 573-9. [http://dx.doi.org/10.1038/hr.2014.43] [PMID: 24621467]

[23] Bhandari SK, Shi J, Molnar MZ, et al. Comparisons of sleep apnoea rate and outcomes among patients with resistant and non-resistant hypertension. Respirology 2016; 21(8): 1486-92. [http://dx.doi.org/10.1111/resp.12840] [PMID: 27427469]

[24] Cuspidi C, Meani S, Fusi V, et al. Is the nocturnal fall in blood pressure reduced in essential hypertensive patients with metabolic syndrome? Blood Press 2004; 13(4): 230-5.

[http://dx.doi.org/10.1080/08037050410021540] [PMID: 15581337]

[25] Hla KM, Young T, Finn L, Peppard PE, Szklo-Coxe M, Stubbs M. Longitudinal association of sleep-disordered breathing and nondipping of nocturnal blood pressure in the Wisconsin Sleep Cohort Study. Sleep 2008; 31(6): 795-800. [http://dx.doi.org/10.1093/sleep/31.6.795] [PMID: 18548823]

[26] Jankowski P, Bednarek A, Olszanecka A, Windak A, Kawecka-Jaszcz K, Czarnecka D. Twenty-four-hour profile of central blood pressure and central-to-peripheral systolic pressure amplification. Am J Hypertens 2013; 26(1): 27-33. [http://dx.doi.org/10.1093/ajh/hps030] [PMID: 23382324]

[27] Williams B, Lacy PS, Thom SM, et al. Differential impact of blood pressure-lowering drugs on central aortic pressure and clinical outcomes: Principal results of the Conduit Artery Function Evaluation (CAFE) study. Circulation 2006; 113(9): 1213-25. [http://dx.doi.org/10.1161/CIRCULATIONAHA.105.595496] [PMID: 16476843]

[28] Bielecka-Dabrowa A, Michalska-Kasiczak M, Gluba A, et al. Biomarkers and echocardiographic predictors of myocardial dysfunction in patients with hypertension. Sci Rep 2015; 5: 8916. [http://dx.doi.org/10.1038/srep08916] [PMID: 25747153]

[29] Padberg JS, Wiesinger A, di Marco GS, et al. Damage of the endothelial glycocalyx in chronic kidney disease. Atherosclerosis 2014; 234(2): $335-43$.

[http://dx.doi.org/10.1016/j.atherosclerosis.2014.03.016] [PMID: 24727235]

[30] Kohara K, Nishida W, Maguchi M, Hiwada K. Autonomic nervous function in non-dipper essential hypertensive subjects. Evaluation by power spectral analysis of heart rate variability. Hypertension 1995; 26(5): 808-14. [http://dx.doi.org/10.1161/01.HYP.26.5.808] [PMID: 7591022]

[31] Satoh M, Hosaka M, Asayama K, et al. Aldosterone-to-renin ratio and nocturnal blood pressure decline assessed by self-measurement of blood pressure at home: The Ohasama Study. Clin Exp Hypertens 2014; 36(2): 108-14. [http://dx.doi.org/10.3109/10641963.2014.892121] [PMID: 24625338]

[32] Graffe CC, Bech JN, Pedersen EB. Effect of high and low sodium intake on urinary aquaporin-2 excretion in healthy humans. Am J Physiol Renal Physiol 2012; 302(2): F264-75.

[http://dx.doi.org/10.1152/ajprenal.00442.2010] [PMID: 21993890]

[33] Jensen JM, Mose FH, Bech JN, et al. Effect of volume expansion with hypertonic- and isotonic saline and isotonic glucose on sodium and water transport in the principal cells in the kidney. BMC Nephrol 2013; 14(202): 2369-14.

[34] Lauridsen TG, Vase H, Bech JN, Nielsen S, Pedersen EB. Direct effect of methylprednisolone on renal sodium and water transport via the principal cells in the kidney. Eur J Endocrinol 2010; 162(5): 961-9. 
[http://dx.doi.org/10.1530/EJE-10-0030] [PMID: 20203161]

[35] Pedersen RS, Bentzen H, Bech JN, Pedersen EB. Effect of water deprivation and hypertonic saline infusion on urinary AQP2 excretion in healthy humans. Am J Physiol Renal Physiol 2001; 280(5): F860-7.

[http://dx.doi.org/10.1152/ajprenal.2001.280.5.F860] [PMID: 11292629]

(C) 2018 Hornstrup et al.

This is an open access article distributed under the terms of the Creative Commons Attribution 4.0 International Public License (CC-BY 4.0), a copy of which is available at: (https://creativecommons.org/licenses/by/4.0/legalcode). This license permits unrestricted use, distribution, and reproduction in any medium, provided the original author and source are credited. 